\title{
Development of Fire Fighting Robot (QRob)
}

\author{
Mohd Aliff ${ }^{1}$, MI Yusof ${ }^{3}$ \\ Malaysian Institute of Industrial \\ Technology \\ Universiti Kuala Lumpur \\ Johor, Malaysia
}

\author{
Nor Samsiah Sani ${ }^{2}$ \\ Center for Artificial Intelligence \\ Technology (CAIT) \\ Universiti Kebangsaan Malaysia \\ Selangor, Malaysia
}

\author{
Azavitra Zainal ${ }^{4}$ \\ Malaysian Institute of Industrial \\ Technology \\ Universiti Kuala Lumpur \\ Johor, Malaysia
}

\begin{abstract}
Fire incident is a disaster that can potentially cause the loss of life, property damage and permanent disability to the affected victim. They can also suffer from prolonged psychological and trauma. Fire fighters are primarily tasked to handle fire incidents, but they are often exposed to higher risks when extinguishing fire, especially in hazardous environments such as in nuclear power plant, petroleum refineries and gas tanks. They are also faced with other difficulties, particularly if fire occurs in narrow and restricted places, as it is necessary to explore the ruins of buildings and obstacles to extinguish the fire and save the victim. With high barriers and risks in fire extinguishment operations, technological innovations can be utilized to assist firefighting. Therefore, this paper presents the development of a firefighting robot dubbed QRob that can extinguish fire without the need for fire fighters to be exposed to unnecessary danger. QRob is designed to be compact in size than other conventional fire-fighting robot in order to ease small location entry for deeper reach of extinguishing fire in narrow space. QRob is also equipped with an ultrasonic sensor to avoid it from hitting any obstacle and surrounding objects, while a flame sensor is attached for fire detection. This resulted in QRob demonstrating capabilities of identifying fire locations automatically and ability to extinguish fire remotely at particular distance. QRob is programmed to find the fire location and stop at maximum distance of $40 \mathrm{~cm}$ from the fire. A human operator can monitor the robot by using camera which connects to a smartphone or remote devices.
\end{abstract}

Keywords-Firefighting robot; compact size robot; ultrasonic sensor; flame sensor; remote control

\section{INTRODUCTION}

A robot is an automated device which performs functions usually attributed to humans or machines tasked with repetitive or flexible set of actions. Numerous studies have shown that robot can be beneficial in medicine [1], rehabilitation [2-6], rescue operation [7, 8] and industry [9]. Over the years, robotics has been introduced in various industries. The industrial robots are multi-function manipulators designed for more specialized materials, divisions, gadgets or devices through various programmatic movements to perform various tasks [10]. In line with the Fourth Industrial Revolution (4IR), there is demand for a one system that can control, communicate and integrate different robots regardless of their types and specifications. Machine learning has also heated up interest in robotics, although only a portion of recent development in robotics can be associated with machine learning. Recent robotic development project has embedded machine learning algorithms [11-15] to increase the intelligence in robots. This will increase the productivity in industry while reducing the cost and electronic waste in a long run.

Studies on the use of humanoid robots are actively carried out to minimize firefighters' injuries and deaths as well as increasing productivity, safety, efficiency and quality of the task given [16]. Robot can be divided into several groups such as Tele-robots, Telepresence robots, Mobile robots, Autonomous robots and Androids robots. Telepresence robot are similar to a tele-robot with the main difference of providing feedback from video, sound and other data. Hence, tele-presence robots are widely used in many fields requiring monitoring capability, such as in child nursery and education, and on improving older adult's social and daily activities [17, 18]. Mobile robot is designed to navigate and carry out tasks with the intervention of human beings [19, 20]. Meanwhile, autonomous robots can perform the task independently and receive the power from the environment, as opposed to android robots which are built to mimic humans [21].

In this paper, a firefighting robot is proposed. The main function of this robot is to become an unmanned support vehicle, developed to search and extinguish fire. There are several existing types of vehicles for firefighting at home and extinguish forest fires [22]. Our proposed robot is designed to be able to work on its own or be controlled remotely. By using such robots, fire identification and rescue activities can be done with higher security without placing fire fighters at high risk and dangerous conditions. In other words, robots can reduce the need for fire fighters to get into dangerous situations. Additionally, having a compact size and automatic control also allows the robot to be used when fire occurs in small and narrow spaces with hazardous environments such as tunnels or nuclear power plants [23, 24].

Thermite and FireRob are two current available fire fighter robots that have been used widely in industry. Thermite (produced by Howe and Howe Technologies Inc) is a firefighting robot that uses a remote control and can operate as far as $400 \mathrm{~m}$. It can deliver up to $1200 \mathrm{gpm}$ of water or $150 \mathrm{psi}$ of foam. The size of this robot is $187.96 \mathrm{~cm}$ x $88.9 \mathrm{~cm}$ x 139.7 $\mathrm{cm}$. This robot powers up to $25 \mathrm{bhp}(18.64 \mathrm{~kW})$ using a diesel engine. The main component in the design of this robot are multi-directional nozzle that is backed by a pump that can deliver $600 \mathrm{gpm}(2271.25 \mathrm{l} / \mathrm{min})$. This robot is designed for use in extreme danger areas, such as planes fires, processing factories, chemical plants or nuclear reactors [25].

FireRob (Manufactured by Croatian manufacturer DOKING) is a fire-fighting vehicle controlled by a single operator 
via remote control. It extinguishes fire without intervention of fire fighters with a high pressure on a hydraulic arm that pumps water up to $55 \mathrm{~m}$ away. It also can carry 1800 litre of water and 600 litre of foam in its two on board tanks. The coating on FireRob allows it to withstand critical temperature of $250^{\circ} \mathrm{C}$ and thermal radiation of $23 \mathrm{~kW} / \mathrm{m}$ for a period of 30 minutes.

In this study, a compact and small firefighter robot has been developed. This robot is named QRob, which is short form of Rescue Robot. This robot can evade obstacles, search and extinguish fire. Furthermore, this robot can increase the productivity, safety, efficiency and quality of the task given. QRob is more compact and more flexible compared to Thermite and FireRob robot. Another advantage of QRob is in its ability to enter location with small entrance or narrow space.

\section{Methodology}

The methodology is divided into three parts. The first part is on the mechanicals schematics, followed by hardware description and the finally on the programming design. All parts were assembled together and experiments were then performed to determine the optimal distance of QRob to extinguish the fire were carried out.

\section{A. Mechanical Design Structure}

Google SketchUp software and AutoCad were used to produce 3D and 2D schematic diagram.

For the main structure of the robot, to get the preferred movement and speed, QRob have two wheels at rear side and two wheels at front side. The wheels have the ability to stabilize the robot and make rotation until 360 degrees. The body of the robot is made from acrylic plate to protect the electronic circuit. The acrylic sheet is resistant to heat of up to $200{ }^{\circ} \mathrm{C}$. This gives the ability to use and work with (cut and drill). The body of acrylic chassis contains holes that make it easier to mounting of various type of sensors and other mechanical components.

The ultrasonic sensor and flame sensor were installed at front of the robot to avoid hitting any obstacles and to detect the fire respectively. In addition, mini camera was installed in front side of the robot to monitor the way and condition of the location and is linked to the smart phone. The structure of fire distinguisher robot is shown in Fig. 1 and Fig. 2.

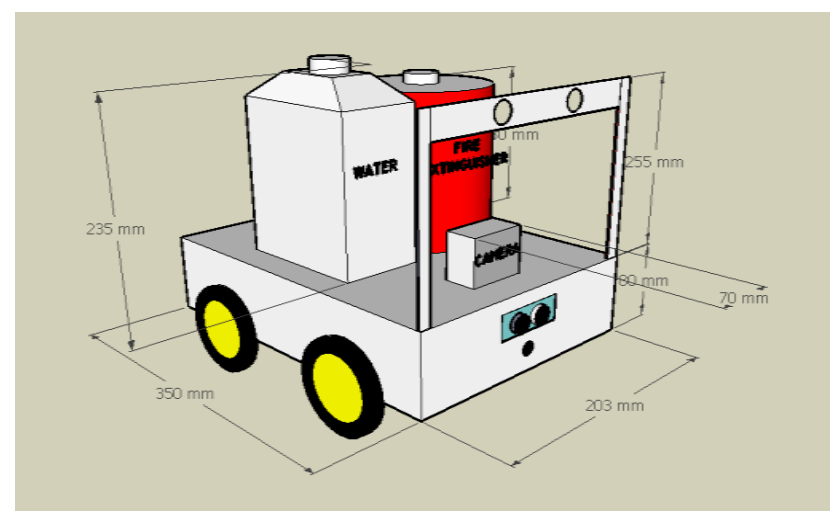

Fig. 1. 3D Structure of QRob with Dimension.

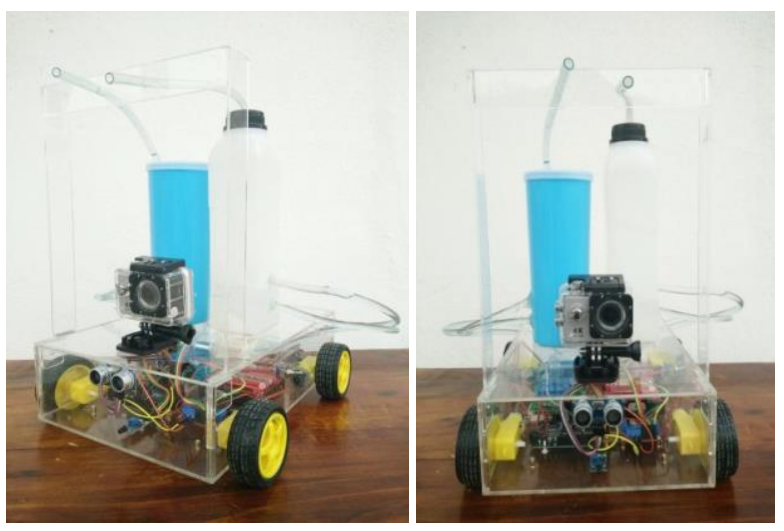

Fig. 2. Firefighting Robot (QRob).

\section{B. Hardware Implementation}

The electronic part is one of the vital parts in the development of QRob. It includes the several types of sensors, microcontroller, DC motor with wheel, Transmitter and Remote control and Water pump. Fig. 3 shows the block diagram of the QRob operation which consists of flame sensor and ultrasonic sensor as input of the system. Arduino Uno is used as a microcontroller that connected with other components. Motor Driver (L298N) is used to activate the moving of the gear motor while Transmitter Remote Control will give output of the system. Flow of water and fire extinguisher were pump after being controlled by the operator. On the other hand, the operator can monitor the robot movements by using camera (Go Pro) which connects to a smartphone.

1) Flame sensor: In most firefighting robots, fire sensors perform an essential part in investigations, which are always used as robot eyes to discover sources of fire [1]. It can be utilized to identify fire based on wavelength of the light at 760 $\mathrm{nm}$ to $1100 \mathrm{~nm}$. The detection angle and distance are roughly 60 degrees and distance $20 \mathrm{~cm}(4.8 \mathrm{~V})$ to $100 \mathrm{~cm}$ (1V) respectively. Flame sensor has two signal pins that are Digital Output (DO) and Analog Output (AO). DO pins will give two kind of information that it's has flame or non-flame while AO pins will detect exact wavelength of different light.

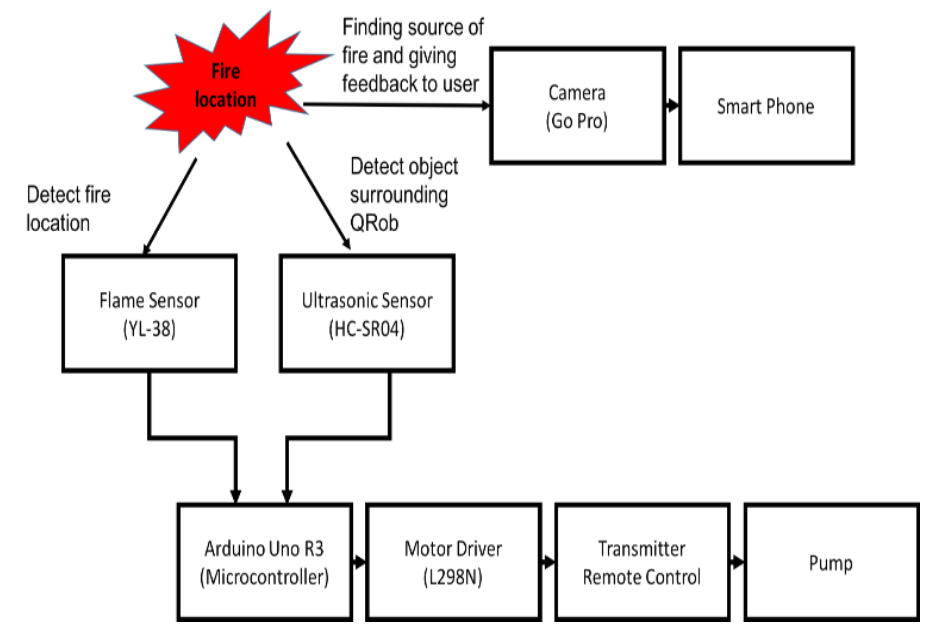

Fig. 3. Block Diagram of QRob. 
2) Ultrasonic sensor: One of the most crucial aspect in inventing an autonomous target detection robot is a barrier and obstacle avoidance. A sensor must be compact, low cost, simple to produce and functional on a larger scale. Moreover, it should be able to sense things with enough limits to let robots to react and travel appropriately.The existing sensors that suit all these requirements are ultrasonic sensors. The HCSR04 ultrasonic sensor is utilized in this study to determine the distance within the range of $2 \mathrm{~cm}$ to $400 \mathrm{~cm}$ with an angle 15 degrees. This sensor transmits waves into the air and receive reflected waves from the object. It has four output pin such as reference voltage (VCC) (operate around $5 \mathrm{~V}$ ), ground pin (GND), digital output (DO) and analog output (AO).

3) DC motor with wheel: DC geared motor with rubber wheel are suitable material for this project. This DC motor are suitable to replace $2 \mathrm{WD}$ and $4 \mathrm{WD}$ car chassis. The working voltage for $\mathrm{DC}$ motor is around $5 \mathrm{~V}$ to $10 \mathrm{~V}$ DC. While the ratio of the gear is $48: 1$. Suitable current for this motor is 73.2 $\mathrm{mA}$. DC motor is used to move the robot to the fire location.

4) Water pump: The water pump is important part in this robot as it will pump water or soap to extinguish the fire depending on the class of fire that occurs. Small-size and light-weight category of water pump has been selected for use in this project.

Moreover, it has low noise, high effectiveness and minimal power consumption. The optimal voltage for this water pump is $6 \mathrm{~V}$. Working voltage for this water pump is around $4 \mathrm{~V}$ to $12 \mathrm{~V}$ with the working current $0.8 \mathrm{~A}$.

5) Transmitter and remote control: In this study, the wireless remote control transmitter and receiver with 4 control modes will be used. Model number of this receiver or remote is $\mathrm{S} 4 \mathrm{C}-\mathrm{AC} 110$. This remote have four buttons. The operating voltage for this remote control is AC $100-120 \mathrm{~V}$, while the working voltage range of relay are AC $110-240 \mathrm{~V}$ or DC $0-$ $28 \mathrm{~V}$. The model number of the transmitter is $\mathrm{C}-4$. The distance of the remote control is $100 \mathrm{~m}$ or $300 \mathrm{ft}$. Power supply for this transmitter are $12 \mathrm{~V}$. The transmitting frequency is $315 \mathrm{MHz} / 433 \mathrm{MHz}$. By utilizing the transmitter and remote control, QRob can be controlled from distant places where the operator who controls it will be in a safe place while the robot will enter into a dangerous fire area.

\section{CONTROL PROGRAMMING}

Fig. 4 shows the relationship between QRob coordinate plane with main surface plane.

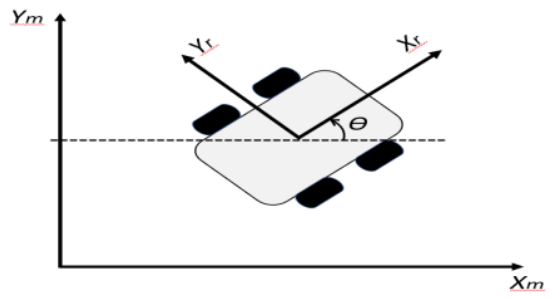

Fig. 4. Relationship between QRob Coordinate Plane with Main Surface Plane.
At first, QRob is originally assumed to be at the center position with the coordinate point at the moment considered as $(0,0)$. When the rotation takes place on the z-axis, as shown as the $\theta$ in Fig. 4, the position change from the original to the final position gives a new coordinate to QRob and is considered as $(\mathrm{x}, \mathrm{y})$ on the coordinate plane. $\left(X_{m}, Y_{m}\right)$ in the figure is the main surface coordinate plane and $\left(X_{r}, Y_{r}\right)$ is the QRob coordinate plane.

$\left[\begin{array}{lll}x^{\prime} & y^{\prime} & \theta^{\prime}\end{array}\right]=[\cos \theta \sin \theta 0] v+\left[\begin{array}{lll}0 & 0 & 1\end{array}\right] \omega$

Equation (1) is to determine the coordinate and angular position for the QRob, where $x^{\prime}, y^{\prime}$ and $\theta^{\prime}$ are the coordinates regarding to the main surface plane $\left(X_{m}, Y_{m}\right)$ and $v$ and $\omega$ are the driving and turning velocity with respect to the coordinates regarding to the QRob coordinate plane $\left(X_{r}, Y_{r}\right)$. Then, adopting differential drive vehicle as the kinematic model of QRob resulting,

$v=\frac{r\left(\omega_{R}+\omega_{L}\right)}{2}$

$\omega=\frac{r\left(\omega_{R}+\omega_{L}\right)}{d}$

where $r$ is radius of the wheel and $d$ is the distance of instantaneous center of rotation.

All the data from sensor is monitored and controlled by Arduino. Fig. 5 shows the Arduino program which all the input and output pin in Arduino need to be declared. Fig. 6 shows the forward and reverse code movement for QRob to find the fire location. Fig. 7 shows the flowchart of Fire Fighting Robot (QRob) using ultrasonic sensor and flame sensor. These codes will be used to program the movement of QRob to find the fire location.

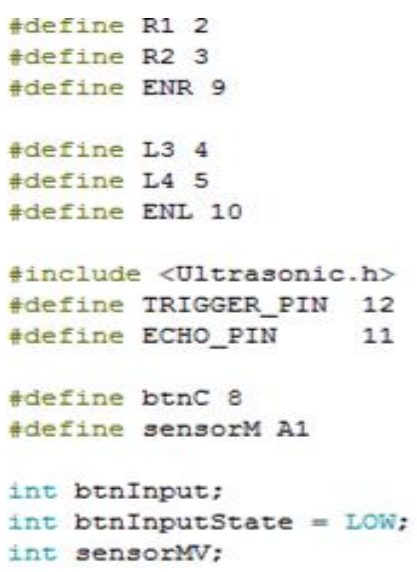

Fig. 5. Declaration Code.

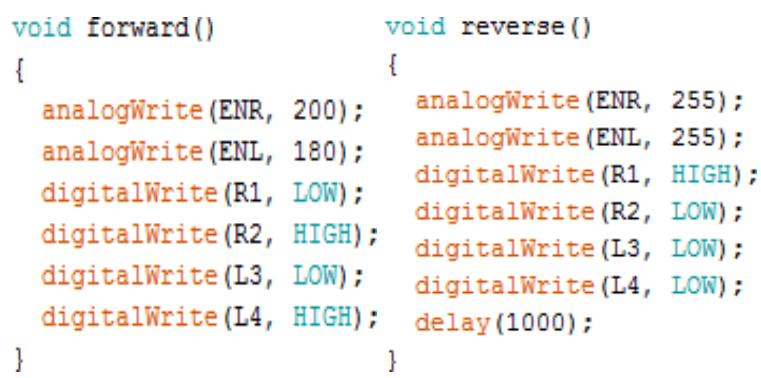

Fig. 6. Forward and Reverse Code Movement. 


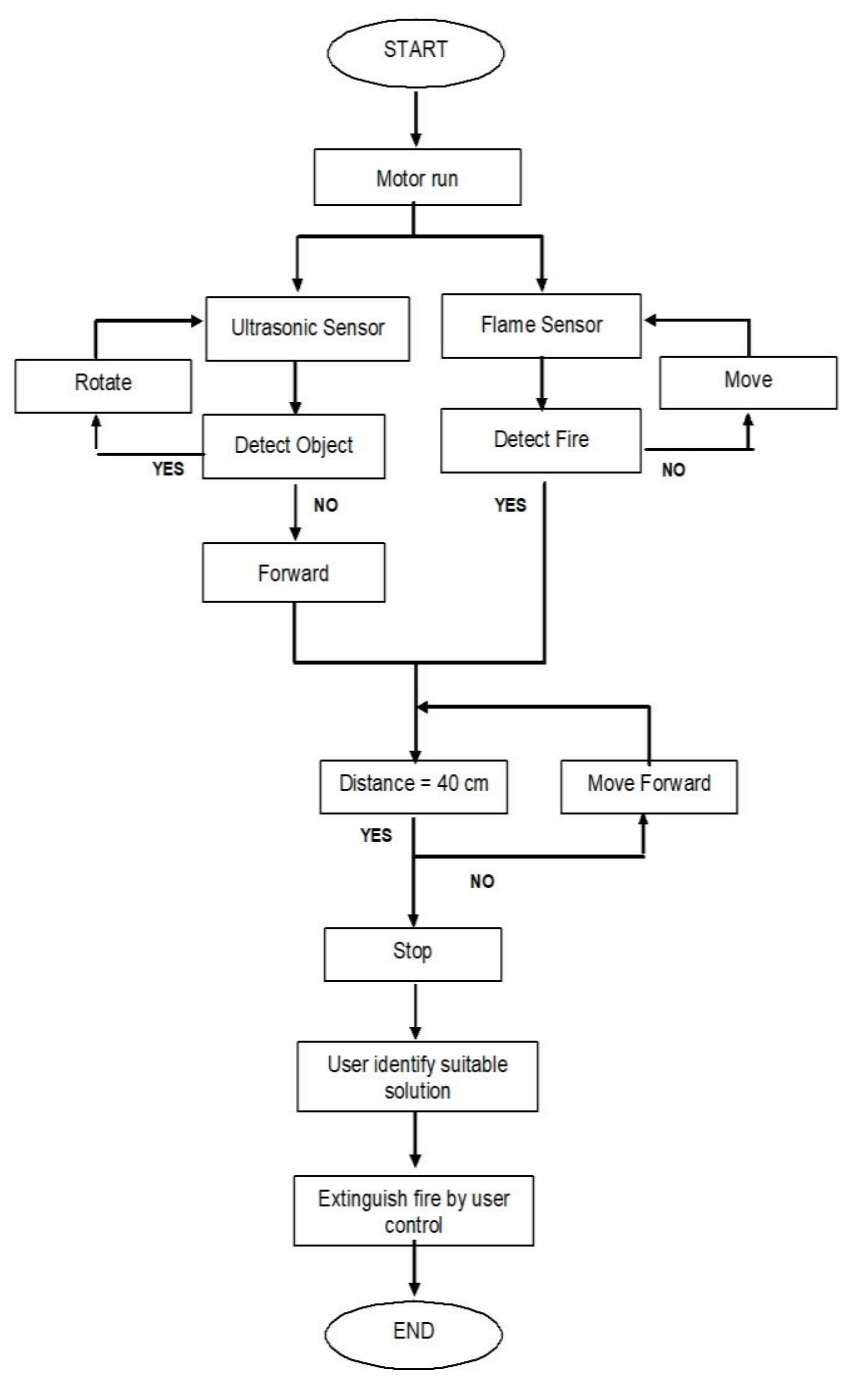

Fig. 7. Flowchart of Fire Fighting Robot (QRob).

\section{RESULT}

Firefighting robot (QRob) has been developed to find the location of fire and extinguish it. QRob has an ability to find the location by using flame sensor and ultrasonic sensor. The flame sensor is functioning to sense the location of fire while ultrasonic sensor is functioning to detect the presence of object around the QRob. Both sensors are connected to Arduino Uno, which controlled the movement of DC motor.

When flame sensor found the fire, the DC motor will stop at $40 \mathrm{~cm}$ from the fire. The operator will be extinguishing the fire using remote control from the distance. The operator also can monitor the QRob by using camera that connects to a smartphone.

\section{A. Time to Extinguish the Fire Depends on Distance of Qrob with Fire Source}

QRob successfully find fire location automatically and extinguish it by operator control. The operator can monitor the location of fire by camera that is connected to the smartphone. Fig. 8 shows the time to extinguish fire depends on distance between QRob and fire, and Fig. 9 shows the image during the fire extinguishing process.

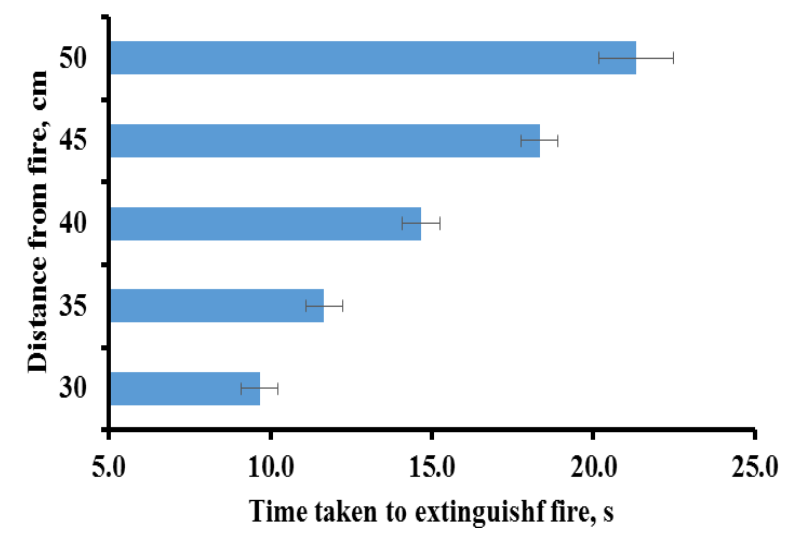

Fig. 8. Time to Extinguish Fire Depends on Distance of QRob with Fire.

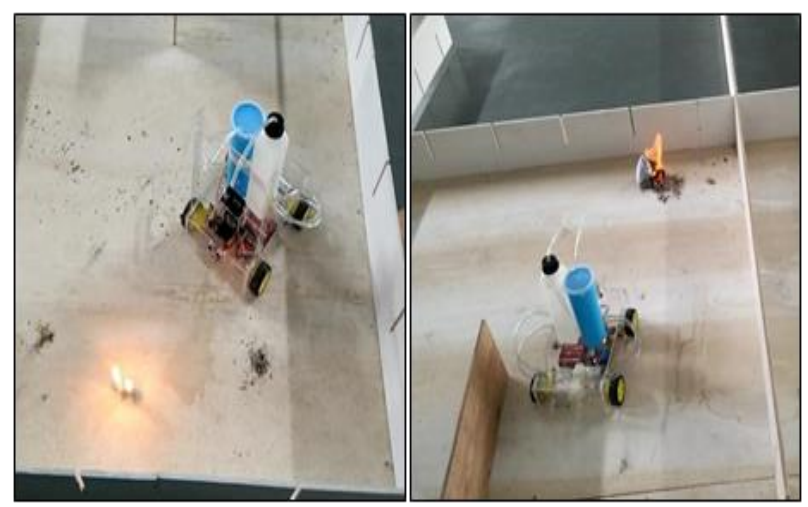

Fig. 9. Distance between QRob and Fire.

From the experimental results, it can be seen that when the distance between QRob and fire is greater, the longer it takes to extinguish the fire. For future planning, it is needed to determine the optimal distance between QRob and fire. This is because to prevent QRob being too close to the fire and at the same time can extinguish the fire in a short time.

\section{B. Ability of QRob to Find Fire Location at Different Distance Route}

QRob is equipped with ultrasonic sensor that allows it to avoid obstacles surround it. Thus, the maze has been designed to test whether the sensor works well and can avoids the barrier as shown in Fig. 10.

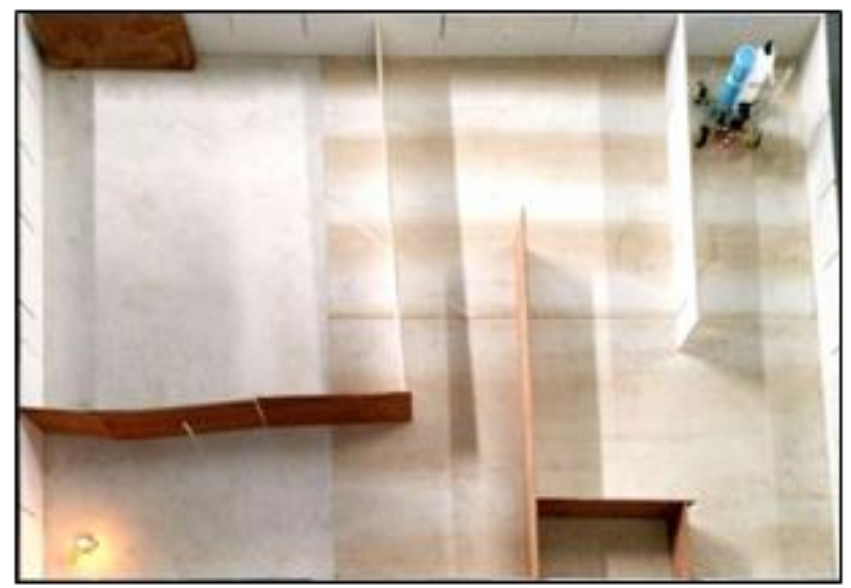

Fig. 10. Maze for Experiment. 


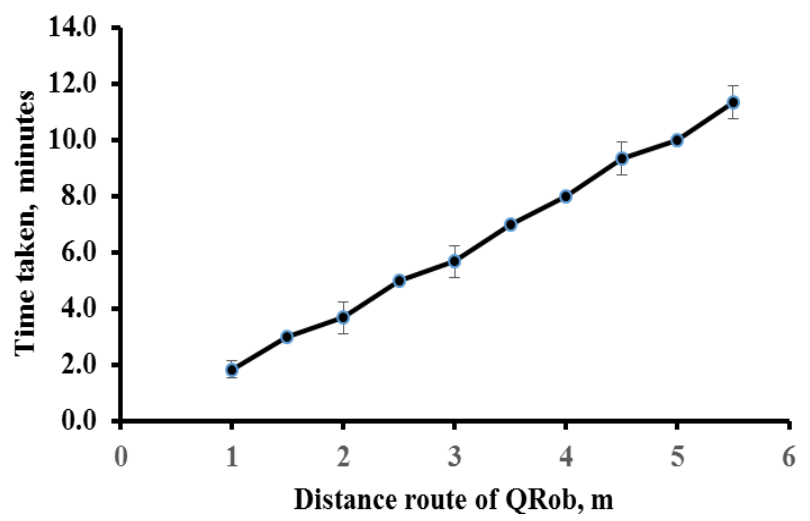

Fig. 11. Time Taken Depends on Distance Route of QRob.
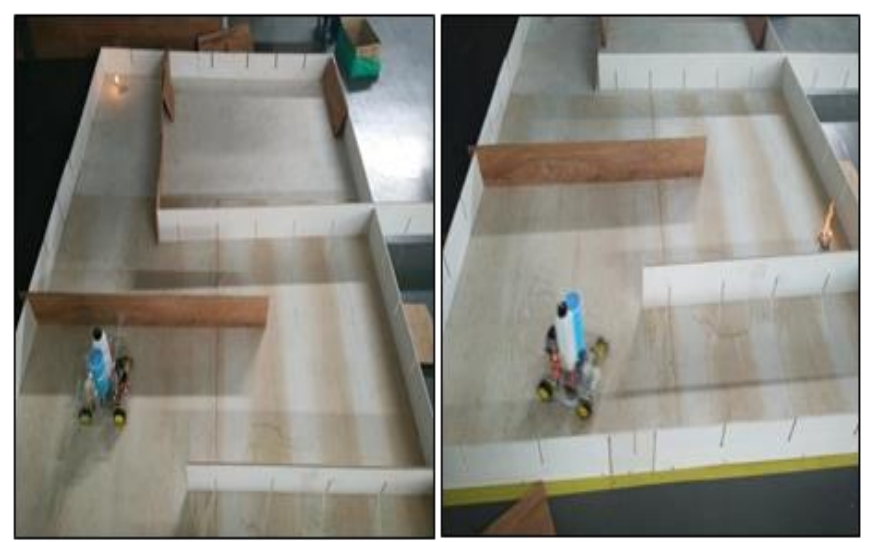

Fig. 12. Different Route Travelled by QRob to Fire Location.

Fig. 11 indicates the time required to arrive at the fire location is depend on the distance route of QRob. From the experimental results, time taken to arrive at fire location is directly proportional to the distance route of QRob. Fig. 12 shows that different fire location placed during the experiment.

\section{DISCUSSION}

This project focuses on the development of firefighting robot (QRob). In this section, the robot control sequence will be discussed. From the results, this project is successfully achieved which are:

\section{1) Flame Sensor Connection}

a) The QRob will not react when the sensor not activate and the QRob will react when the sensor activates as well.

b) This sensor is connecting with DC motor.

c) This sensor OFF when fire was not detected and DC motor and Ultrasonic Sensor ON.

d) This sensor ON when fire is detected, then DC motor and Ultrasonic Sensor will automatically OFF.

When Flame Sensor $=1$; DC Motor $=0$,

Ultrasonic Sensor $=0$.

When Flame Sensor $=0$; DC Motor $=1$,

Ultrasonic Sensor $=1$.
2) Ultrasonic Sensor Connection

a) This sensor will detect the object of their surroundings.

b) This sensor $\mathrm{ON}$ when DC motor ON but Flame Sensor will OFF.

c) This sensor OFF when Flame sensor ON.

When Ultrasonic Sensor $=1$; DC Motor $=1$,

Flame Sensor $=0$,

When Ultrasonic Sensor $=0$; DC Motor $=0$,

Flame Sensor $=1$,

\section{3) DC Motor}

a) This Motor is connecting with driver motor and Arduino Uno.

b) This Motor ON when Ultrasonic Sensor ON, Flame Sensor OFF.

c) This Motor OFF when Flame Sensor ON.

When DC Motor = 1; Ultrasonic Sensor $=1$,

Flame Sensor $=0$,

When DC Motor $=0$; Ultrasonic Sensor $=0$,

Flame Sensor $=1$,

\section{CONCLUSIONS}

Overall, a fire-fighting robot that can be controlled from some distance has been successfully developed. It has advantageous features such as ability to detect location of fire automatically besides having a compact body and lightweight structure. QRob also has the ability to avoid hitting any obstacle or surrounding objects due to its provision of an ultrasonic sensor. The QRob robot can be used at a place that has a small entrance or in small spaces because it has a compact structure. The operator is able to extinguish fire using remote control from longer distance. Operators can also monitor the environmental conditions during the process of firefighting by using the camera that is connected to the smartphone. From the experimental results, the robot can sense smokes and fire accurately in a short time. As a conclusion, the project entitled "Development of Fire Fighting Robot (QRob)" has achieved its aim and objective successfully.

\section{REFERENCES}

[1] Jeelani, S., et al., Robotics and medicine: A scientific rainbow in hospital. Journal of Pharmacy \& Bioallied Sciences, 2015. 7(Suppl 2): p. S381-S383.

[2] Aliff, M., S. Dohta, and T. Akagi, Simple Trajectory Control Method of Robot Arm Using Flexible Pneumatic Cylinders. Journal of Robotics and Mechatronics, 2015. 27(6): p. 698-705.

[3] Aliff M, D.S., and Akagi T, Control and analysis of simple-structured robot arm using flexible pneumatic cylinders. International Journal of Advanced and Applied Sciences, 2017. 4(12): p. 151-157.

[4] Aliff, M., S. Dohta, and T. Akagi, Control and analysis of robot arm using flexible pneumatic cylinder. Mechanical Engineering Journal, 2014. 1(5): p. DR0051-DR0051.

[5] M. Aliff, S. Dohta and T. Akagi, Trajectory controls and its analysis for robot arm using flexible pneumatic cylinders," IEEE International Symposium on Robotics and Intelligent Sensors (IRIS), 2015, pp. 48-54.

[6] M. Aliff, S. Dohta and T. Akagi, Trajectory control of robot arm using flexible pneumatic cylinders and embedded controller, IEEE 
International Conference on Advanced Intelligent Mechatronics (AIM), 2015, pp. 1120-1125.

[7] C. Xin, D. Qiao, S. Hongjie, L. Chunhe and Z. Haikuan, Design and Implementation of Debris Search and Rescue Robot System Based on Internet of Things, International Conference on Smart Grid and Electrical Automation (ICSGEA), 2018, pp. 303-307.

[8] Yusof, M., and Dodd, T., Pangolin: A Variable Geometry Tracked Vehicle With Independent Track Control, Field Robotics, pp. 917-924.

[9] Day, C.-P., Robotics in Industry-Their Role in Intelligent Manufacturing. Engineering, 2018. 4(4): p. 440-445.

[10] J. Lee, G. Park, J. Shin and J. Woo, Industrial robot calibration method using denavit - Hatenberg parameters, 17th International Conference on Control, Automation and Systems (ICCAS), 2017, pp. 1834-1837.

[11] Sani, N. S., Shamsuddin, I. I. S., Sahran, S., Rahman, A. H. A and Muzaffar, E. N, Redefining selection of features and classification algorithms for room occupancy detection, International Journal on Advanced Science, Engineering and Information Technology, 2018, 8(42), pp. 1486-1493.

[12] Holliday, J. D., Sani, N., and Willett, P., Calculation of substructural analysis weights using a genetic algorithm, Journal of Chemical Information and Modeling, 2015, 55(2), pp. 214-221.

[13] Holliday, J. D., N. Sani, and P. Willett, Ligand-based virtual screening using a genetic algorithm with data fusion, Match: Communications in Mathematical and in Computer Chemistry, 80, pp. 623-638.

[14] SamsiahSani, N., Shlash, I., Hassan, M., Hadi, A., and Aliff, M, Enhancing malaysia rainfall prediction using classification techniques, J. Appl. Environ. Biol. Sci, 2017, 7(2S), pp. 20-29.

[15] Sani, N.S., Rahman, M.A., Bakar, A.A., Sahran, S. and Sarim, H.M, Machine learning approach for bottom 40 percent households (B40) poverty classification, International Journal on Advanced Science, Engineering and Information Technology, 2018, 8(4-2), pp.1698-1705.

[16] Kim, J.-H., S. Jo, and B.Y. Lattimer, Feature Selection for Intelligent Firefighting Robot Classification of Fire, Smoke, and Thermal Reflections Using Thermal Infrared Images. Journal of Sensors, 2016. 2016: p. 13.
[17] Tanaka, F., et al., Telepresence robot helps children in communicating with teachers who speak a different language, in Proceedings of the 2014 ACM/IEEE international conference on Human-robot interaction. 2014, ACM: Bielefeld, Germany. p. 399-406.

[18] J. Ahn and G. J. Kim, Remote collaboration using a tele-presence mobile projector robot tele-operated by a smartphone, IEEE/SICE International Symposium on System Integration (SII), 2016, pp. 236241.

[19] Harik, E.H. and A. Korsaeth, Combining Hector SLAM and Artificial Potential Field for Autonomous Navigation Inside a Greenhouse. Robotics, 2018. 7(2): p. 22.

[20] Acosta Calderon, C.A., E.R. Mohan, and B.S. Ng, Development of a hospital mobile platform for logistics tasks. Digital Communications and Networks, 2015. 1(2): p. 102-111.

[21] H. Hyung, B. Ahn, B. Cruz and D. Lee, Analysis of android robot lipsync factors affecting communication, 11th ACM/IEEE International Conference on Human-Robot Interaction (HRI), 2016, pp. 441-442.

[22] R. N. Haksar and M. Schwager, Distributed Deep Reinforcement Learning for Fighting Forest Fires with a Network of Aerial Robots, IEEE/RSJ International Conference on Intelligent Robots and Systems (IROS), 2018, pp. 1067-1074.

[23] J. Raju, S. S. Mohammed, J. V. Paul, G. A. John and D. S. Nair, Development and implementation of arduino microcontroller based dual mode fire extinguishing robot, IEEE International Conference on Intelligent Techniques in Control, Optimization and Signal Processing (INCOS), 2017, pp. 1-4.

[24] Tushar Nandkishor Satbhai, R.M.K., Anant Vijay Patil, Manish Patil, Fire Fighting Robot. International Journal on Recent and Innovation Trends in Computing and Communication (IJRITCC), 2016. 4(4): p. 799-803.

[25] Nuţă, I., O. Orban, and L. Grigore, Development and Improvement of Technology in Emergency Response. Procedia Economics and Finance, 2015. 32: p. 603-609. 